\title{
Existence and uniqueness of solutions of Hahn difference equations
}

\author{
Alaa E Hamza ${ }^{1 *}$ and Samah M Ahmed ${ }^{2}$
}

"Correspondence:

hamzaaeg2003@yahoo.com

1 Department of Mathematics,

Faculty of Science, Cairo University,

Giza, Egypt

Full list of author information is

available at the end of the article

\begin{abstract}
Hahn introduced the difference operator $D_{q, \omega} f(t)=(f(q t+\omega)-f(t)) /(t(q-1)+\omega)$ in 1949 , where $0<q<1$ and $\omega>0$ are fixed real numbers. This operator extends the classical difference operator $\Delta_{\omega} f(t)=(f(t+\omega)-f(t)) / \omega$ as the Jackson $q$-difference operator $D_{q} f(t)=(f(q t)-f(t)) /(t(q-1))$.

In this paper, we present new results of the calculus based on the Hahn difference operator. Also, we establish an existence and uniqueness result of solutions of Hahn difference equations by using the method of successive approximations.
\end{abstract}

Keywords: Hahn difference operator; Jackson q-difference operator

\section{Introduction and preliminaries}

Hahn introduced his difference operator which is defined by

$$
D_{q, \omega} f(t)=\frac{f(q t+\omega)-f(t)}{t(q-1)+\omega}
$$

at $t \neq \omega /(1-q)$ and the usual derivative at $t=\omega /(1-q)$, where $0<q<1$ and $\omega>0$ are fixed real numbers $[1,2]$. This operator unifies and generalizes two well-known difference operators. The first is Jackson $q$-difference operator defined by

$$
D_{q} f(t)=\frac{f(q t)-f(t)}{t(q-1)}
$$

where $q$ is fixed. Here $f$ is supposed to be defined on a $q$-geometric set $A \subset \mathbb{R}$, for which $q t \in A$ whenever $t \in A$, see [3-10]. The second operator is the forward difference operator

$$
\Delta_{\omega} f(t)=\frac{f(t+\omega)-f(t)}{\omega}
$$

where $\omega>0$ is fixed, see [11-14].

In [15], Annaby et al. gave a rigorous analysis of the calculus associated with $D_{q, \omega}$. They stated and proved some basic properties of such a calculus. For instance, they defined a right inverse of $D_{q, \omega}$ in terms of both the Jackson $q$-integral; see [9], which contains the right inverse of $D_{q}$ and Nörlund sum; $c f$. [13], which involves the right inverse of $\Delta_{\omega}$. Then they proved a fundamental theorem of Hahn's calculus. An essential function which plays an important role in this calculus is $h(t)=q t+\omega$. This function is normally taken to be

\section{Springer}

○2013 Hamza and Ahmed; licensee Springer. This is an Open Access article distributed under the terms of the Creative Commons Attribution License (http://creativecommons.org/licenses/by/2.0), which permits unrestricted use, distribution, and reproduction in any medium, provided the original work is properly cited. 
defined on an interval $I$, which contains the number $\theta=\omega /(1-q)$. One can see that the $k$ th order iteration of $h(t)$ is given by

$$
h^{k}(t)=q^{k} t+\omega[k]_{q}, \quad t \in I .
$$

The sequence $h^{k}(t)$ is uniformly convergent to $\theta$ on $I$. Here $[k]_{q}$ is defined by

$$
[k]_{q}=\frac{1-q^{k}}{1-q} .
$$

Throughout this paper, $I$ is any interval of $\mathbb{R}$ containing $\theta$ and $X$ is a Banach space.

Definition 1.1 Assume that $f: I \rightarrow X$ is a function, and let $a, b \in I$. The $q$, $\omega$-integral of $f$ from $a$ to $b$ is defined by

$$
\int_{a}^{b} f(t) d_{q, \omega} t=\int_{\theta}^{b} f(t) d_{q, \omega} t-\int_{\theta}^{a} f(t) d_{q, \omega} t
$$

where

$$
\int_{\theta}^{x} f(t) d_{q, \omega} t=(x(1-q)-\omega) \sum_{k=0}^{\infty} q^{k} f\left(h^{k}(x)\right), \quad x \in I
$$

provided that the series converges at $x=a$ and $x=b$.

Definition 1.2 [15] For certain $z \in \mathbb{C}$, the $q$, $\omega$-exponential functions $e_{z}(t)$ and $E_{z}(t)$ are defined by

$$
e_{z}(t)=\sum_{k=0}^{\infty} \frac{(z(t(1-q)-\omega))^{k}}{(q ; q)_{k}}=\frac{1}{\prod_{k=0}^{\infty}\left(1-z q^{k}(t(1-q)-\omega)\right)}
$$

and

$$
E_{z}(t)=\sum_{k=0}^{\infty} \frac{q^{\frac{1}{2} k(k-1)}(z(t(1-q)-\omega))^{k}}{(q ; q)_{k}}=\prod_{k=0}^{\infty}\left(1+z q^{k}(t(1-q)-\omega)\right) .
$$

To guarantee the convergence of the infinite product in (1.1) with $t \in \mathbb{C}$, we assume additionally that

$$
|t-\theta|<\frac{1}{|z(1-q)|},
$$

see $[16,17]$. For a fixed $z \in \mathbb{C}$, (1.2) converges for all $t \in \mathbb{C}$, defining an entire function of order zero. For the proofs of the equalities in (1.1) and (1.2), see [18, Section 1.3] and [17]. Here the $q$-shifted factorial $(b ; q)_{n}$ for a complex number $b$ and $n \in \mathbb{N}_{0}$ is defined to be

$$
(b ; q)_{n}= \begin{cases}\prod_{j=1}^{n}\left(1-b q^{j-1}\right), & \text { if } n \in \mathbb{N} \\ 1, & \text { if } n=0\end{cases}
$$

The following results were mentioned in [15], and we need them in this paper. 
Lemma 1.3 Let $f, g: I \rightarrow \mathbb{R}$ be $q, \omega$ differentiable at $t \in I$. Then the following statements are true:

(i) $D_{q, \omega}(f+g)(t)=D_{q, \omega} f(t)+D_{q, \omega} g(t)$,

(ii) $D_{q, \omega}(f g)(t)=D_{q, \omega}(f(t)) g(t)+f(h(t)) D_{q, \omega} g(t)$,

(iii) for any constant $c \in X, D_{q, \omega}(c f)(t)=c D_{q, \omega}(f(t))$,

(iv) $D_{q, \omega}(f / g)(t)=\left(D_{q, \omega}(f(t)) g(t)-f(t) D_{q, \omega} g(t)\right) /(g(t) g(h(t)))$ for $g(t) g(h(t)) \neq 0$.

We notice that (ii) and (iv) are true even iff $: I \rightarrow X$. Also, (i) is true iff, $g: I \rightarrow X$.

Lemma 1.4 For a fixed $z \in \mathbb{C}$, the parametric $q$, $\omega$-exponential functions $e_{z}(t)$ and $E_{-z}(t)$ are the unique solutions of the first order initial value problems

$$
D_{q, \omega} y(t)=z y(t), \quad y(\theta)=1, \quad|t-\theta|<\frac{1}{|z(1-q)|}
$$

and

$$
D_{q, \omega} y(t)=-z y(q t+\omega), \quad y(\theta)=1, \quad z, t \in \mathbb{C},
$$

respectively.

Lemma 1.5 Let $s \in I, s>\theta, f: I \rightarrow \mathbb{R}$ and $g: I \rightarrow \mathbb{R}$ be $q$, $\omega$-integrable on I. If $|f(t)| \leq g(t)$ for all $t \in\left\{s q^{k}+\omega[k]_{q}\right\}_{k=0}^{\infty}$, then for $a, b \in\left\{s q^{k}+\omega[k]_{q}\right\}_{k=0}^{\infty}, a<b$,

$$
\left|\int_{\theta}^{b} f(t) d_{q, \omega} t\right| \leq \int_{\theta}^{b} g(t) d_{q, \omega} t \text { and }\left|\int_{a}^{b} f(t) d_{q, \omega} t\right| \leq \int_{a}^{b} g(t) d_{q, \omega} t .
$$

Theorem 1.6 Let $f: I \rightarrow \mathbb{R}$ be continuous at $\theta$. Define

$$
F(x):=\int_{\theta}^{x} f(t) d_{q, \omega} t, \quad x \in I .
$$

Then $F$ is continuous at $\theta$. Furthermore, $D_{q, \omega} F(x)$ exists for every $x \in I$ and

$$
D_{q, \omega} F(x)=f(x) .
$$

Conversely,

$$
\int_{a}^{b} D_{q, \omega} f(t) d_{q, \omega} t=f(b)-f(a) \quad \forall a, b \in I .
$$

Lemma 1.5 and Theorem 1.6 are also true if $f$ is a function with values in $X$ with replacing the norm $\|\cdot\|$ instead of the modulus $|\cdot|$.

The aim of this paper is to establish an existence and uniqueness result of solutions of the first order abstract Hahn difference equations by using the method of successive approximations. This method is a very powerful tool that dates back to the works of Liouville [19] and Picard [20]. It is based on defining a sequence of functions $\left\{\phi_{k}\right\}_{k=0}^{\infty}$ and showing that $\phi_{k}$ will successively approximate the solution $\phi$ in the sense that the 'error' between the two monotonically decreases as $k$ increases. Also, it differs from fixed point methods 
and topological ideas that were used by some researchers to develop the existence and uniqueness of solutions.

We organize this paper as follows.

In Section 2, we prove Gronwall's and Bernoulli's inequalities with respect to the Hahn difference operator. In Section 3, we establish mean value theorems in the calculus based on this operator. In Sections 4 and 5, we apply the method of successive approximations to obtain the local and global existence and uniqueness theorem of first order Hahn difference equations in Banach spaces. Hence, we deduce this theorem for the $n$th order Hahn difference equations.

\section{Gronwall's and Bernoulli's inequalities}

In this section, $I$ is a subinterval of $\left[\theta-\frac{1}{|r|(1-q)}, \theta+\frac{1}{|r|(1-q)}\right]$, where $r \in \mathbb{R}$, and $f$ is a real valued function defined on $I$.

Theorem 2.1 Assume that $r \in \mathbb{R}$, and $y, f$ are continuous at $\theta$. Then

$$
D_{q, \omega} y(t) \leq r y(t)+f(t)
$$

for all $t \in I$ implies that

$$
y(t) \leq y(\theta) e_{r}(t)+e_{r}(t) \int_{\theta}^{t} E_{-r}(h(\tau)) f(\tau) d_{q, \omega} \tau .
$$

Proof We have

$$
\begin{aligned}
D_{q, \omega}\left[y(t) E_{-r}(t)\right] & =D_{q, \omega} y(t) E_{-r}(h(t))+y(t) D_{q, \omega} E_{-r}(t) \\
& =\left(D_{q, \omega} y(t)\right) E_{-r}(h(t))+y(t)\left(-r E_{-r}(h(t))\right) \\
& =E_{-r}(h(t))\left(D_{q, \omega} y(t)-r y(t)\right) \\
& \leq E_{-r}(h(t)) f(t) .
\end{aligned}
$$

Integrating both sides from $\theta$ to $t$ in the inequality above, we obtain

$$
y(t) E_{-r}(t)-y(\theta) E_{-r}(\theta) \leq \int_{\theta}^{t} E_{-r}(h(\tau)) f(\tau) d_{q, \omega} \tau .
$$

This implies that

$$
y(t) \leq y(\theta) e_{r}(t)+e_{r}(t) \int_{\theta}^{t} E_{-r}(h(\tau)) f(\tau) d_{q, \omega} \tau .
$$

Theorem 2.2 (Gronwall's inequality) Let $r \in \mathbb{R}^{\geq 0}$, and $y, f$ be continuous at $\theta$. Then

$$
y(t) \leq f(t)+\int_{\theta}^{t} r y(\tau) d_{q, \omega} \tau
$$

for all $t \in I$ implies that

$$
y(t) \leq f(t)+r e_{r}(t) \int_{\theta}^{t} E_{-r}(h(\tau)) f(\tau) d_{q, \omega} \tau .
$$


Proof Set $z(t)=\int_{\theta}^{t} r y(\tau) d_{q, \omega} \tau$. Then $z(\theta)=0, D_{q, \omega} z(t)=r y(t) \leq r(f(t)+z(t))$ and $D_{q, \omega} z(t) \leq$ $r f(t)+r z(t)$. Consequently,

$$
z(t) \leq 0+e_{r}(t) \int_{\theta}^{t} r E_{-r}(h(\tau)) f(\tau) d_{q, \omega} \tau
$$

Hence,

$$
y(t) \leq f(t)+r e_{r}(t) \int_{\theta}^{t} E_{-r}(h(\tau)) f(\tau) d_{q, \omega} \tau .
$$

Theorem 2.3 (Bernoulli's inequality) Let $r \in \mathbb{R}$. Then, $e_{r}(t) \geq 1+r(t-\theta)$ for all $t \in[\theta, \theta+$ $\left.\frac{1}{|r|(1-q)}\right]$.

Proof Let $y(t)=r(t-\theta)$. Then,

$$
r y(t)+r=r^{2}(t-\theta)+r \geq r=D_{q, \omega} y(t) .
$$

Since $y(\theta)=0$, we have by Theorem 2.1

$$
\begin{aligned}
y(t) & \leq y(\theta) e_{r}(t)+e_{r}(t) \int_{\theta}^{t} r E_{-r}(h(\tau)) d_{q, \omega} \tau \\
& =e_{r}(t)\left(-\int_{\theta}^{t} D_{q, \omega} E_{-r}(\tau) d_{q, \omega} \tau\right) \\
& =e_{r}(t)\left(1-E_{-r}(t)\right) \\
& =e_{r}(t)-1 .
\end{aligned}
$$

Therefore, $e_{r}(t) \geq 1+r(t-\theta)$.

\section{Mean value theorems}

Theorem 3.1 Let $f: I \rightarrow X, g: I \rightarrow \mathbb{R}$ be $q$, $\omega$ differentiable on $I$ and $s \in I$.

Assume that $\left\|D_{q, \omega} f(t)\right\| \leq D_{q, \omega} g(t)$ for all $t \in\left\{s q^{k}+\omega[k]_{q}\right\}_{k=0}^{\infty}$. Then $\|f(b)-f(a)\| \leq g(b)-$ $g(a)$ for all $a \leq b, a, b \in\left\{s q^{k}+\omega[k]_{q}\right\}_{k=0}^{\infty}$.

Proof The inequality $\left\|\int_{a}^{b} D_{q, \omega} f(t) d_{q, \omega} t\right\| \leq \int_{a}^{b} D_{q, \omega} g(t) d_{q, \omega} t$ implies that $\|f(b)-f(a)\| \leq$ $g(b)-g(a)$ for all $a \leq b, a, b \in\left\{s q^{k}+\omega[k]_{q}\right\}_{k=0}^{\infty}$.

Corollary 3.2 Suppose that $f, g: I \rightarrow X$ is $q$, $\omega$ differentiable. The following statements are true:

(i) For every $s \in I$, the inequality $\|f(b)-f(a)\| \leq \sup _{t \in I}\left\|D_{q, \omega} f(t)\right\|(b-a)$ holds for all $a \leq b, a, b \in\left\{s q^{k}+\omega[k]_{q}\right\}_{k=0}^{\infty}$.

(ii) If $D_{q, \omega} f(t)=0$ for all $t \in I$, then $f$ is a constant function.

(iii) If $D_{q, \omega} f(t)=D_{q, \omega} g(t)$ for all $t \in I$. Then $g(t)=f(t)+c$ for all $t \in I$, where $c$ is a constant in $X$.

Proof (i) Let $g(t)=\sup _{t \in I}\left\|D_{q, \omega} f(t)\right\|(t-\theta)$. Then $D_{q, \omega} g(t)=\sup _{t \in I}\left\|D_{q, \omega} f(t)\right\| \geq\left\|D_{q, \omega} f(t)\right\|$ for all $t \in\left\{s q^{k}+\omega[k]_{q}\right\}_{k=0}^{\infty}$. 
By Theorem 3.1,

$$
\begin{aligned}
\|f(b)-f(a)\| & \leq g(b)-g(a) \\
& =\sup _{t \in I}\left\|D_{q, \omega} f(t)\right\|(b-\theta-a+\theta) \\
& =\sup _{t \in I}\left\|D_{q, \omega} f(t)\right\|(b-a) .
\end{aligned}
$$

(ii) Statement (i) implies that $f\left(s q^{k}+\omega[k]_{q}\right)=f(s)$ for every $s \in I$ and $k=0,1, \ldots$ Letting $k \rightarrow \infty$, we obtain $f(s)=f(\theta)$ for all $s \in I$.

(iii) Can be deduced immediately from (ii).

As a direct consequence of Theorem 1.6, we get the following result.

Theorem 3.3 Let $f: I \rightarrow X$ be continuous at $\theta$. Then

$$
\int_{t}^{h(t)} f(\tau) d_{q, \omega} \tau=(h(t)-t) f(t)
$$

\section{Successive approximations and local results}

Throughout the remainder of the paper,

$$
S\left(x_{0}, b\right)=\left\{x \in X:\left\|x-x_{0}\right\| \leq b\right\}
$$

and $R$ is the rectangle

$$
R=[\theta, \theta+a] \times S\left(x_{0}, b\right),
$$

where $a$ and $b$ are fixed positive numbers.

Theorem 4.1 Assume that $f: R \rightarrow X$ satisfies the following conditions:

(i) $f(t, x)$ is continuous at $t=\theta$ for every $x \in S\left(x_{0}, b\right)$.

(ii) There is a positive constant $A$ such that the following Lipschitz condition $\|f(t, x)-f(t, y)\| \leq A\|x-y\|$ for all $x, y \in X$ is satisfied.

Then there is $h>0$ such that the following Cauchy problem

$$
\left.\begin{array}{l}
D_{q, \omega} x(t)=f(t, x) \\
x(\theta)=x_{0}
\end{array}\right\}
$$

has a unique solution $x(t)$ on $[\theta, \theta+h]$.

Proof Since $f$ is continuous at $t=\theta$, there exists $\gamma>0$ such that $\|f(t, x)-f(\theta, x)\|<1$ for all $t$ with $|t-\theta|<\gamma$. Hence,

$$
\begin{aligned}
\|f(t, x)\| & \leq\|f(t, x)-f(\theta, x)\|+\left\|f(\theta, x)-f\left(\theta, x_{0}\right)\right\|+\left\|f\left(\theta, x_{0}\right)\right\| \\
& <1+A b+\left\|f\left(\theta, x_{0}\right)\right\|
\end{aligned}
$$


for all $t \in I, x \in X$ such that $|t-\theta|<\gamma$ and $\left\|x-x_{0}\right\|<b$. Define the following constants $K$, $h$ to be

$$
\begin{aligned}
& K=\sup _{t \in[\theta, \theta+a], x \in S\left(x_{0}, b\right)}\|f(t, x)\|, \\
& h=\min \left\{\gamma, \frac{1-q}{A}, \frac{b}{K}\right\} .
\end{aligned}
$$

We establish the existence of the solution $\phi(t)$ of (4.1) by the method of successive approximations.

We consider the sequence defined as follows:

$$
\left.\begin{array}{l}
\phi_{0}(t)=x_{0}, \\
\phi_{k+1}(t)=x_{0}+\int_{\theta}^{t} f\left(s, \phi_{k}(s)\right) d_{q, \omega} s, \quad k \geq 0 .
\end{array}\right\}
$$

The proof of the existence consists of four steps.

(i) $\phi_{k}(t) \in S\left(x_{0}, b\right), k \geq 0, t \in I$. Indeed, we have $\left\|\phi_{0}(t)-x_{0}\right\|=0 \leq b$. Assume that the inequality $\left\|\phi_{k}(t)-x_{0}\right\| \leq b$ holds. This implies that

$$
\begin{aligned}
\left\|\phi_{k+1}(t)-x_{0}\right\| & \leq \int_{\theta}^{t}\left\|f\left(s, \phi_{k}(s)\right)\right\| d_{q, \omega} s \\
& \leq K \int_{\theta}^{t} d_{q, \omega} s \\
& \leq b
\end{aligned}
$$

i.e., $\phi_{k}(t) \in S\left(x_{0}, b\right), k \in \mathbb{Z}^{\geq 0}$. Also, each $\phi_{k}(t)$ is continuous at $t=\theta$.

(ii) Now, we show by induction that

$$
\left\|\phi_{m+1}(t)-\phi_{m}(t)\right\| \leq K A^{m} h^{m+1}, \quad t \in[\theta, \theta+h] .
$$

First, $\left\|\phi_{1}(t)-\phi_{0}(t)\right\| \leq K \int_{\theta}^{t} d_{q, \omega} s \leq K h$.

Assume that (4.3) is true. Hence,

$$
\begin{aligned}
\left\|\phi_{m+2}(t)-\phi_{m+1}(t)\right\| & \leq \int_{\theta}^{t}\left\|f\left(s, \phi_{m+1}(s)\right)-f\left(s, \phi_{m}(s)\right)\right\| d_{q, \omega} s \\
& \leq A \int_{\theta}^{t}\left\|\phi_{m+1}(t)-\phi_{m}(t)\right\| d_{q, \omega} s \\
& \leq K A^{m+1} h^{m+1} \int_{\theta}^{t} d_{q, \omega} s \\
& \leq K A^{m+1} h^{m+2} .
\end{aligned}
$$

Thus, inequality (4.3) is true for every $m \in \mathbb{Z}^{\geq 0}$.

(iii) We show that $\phi_{m}(t)$ converges uniformly to a function $\phi(t)$ on $[\theta, \theta+h]$.

We write $\phi_{m}(t)$ as the following sum

$$
\phi_{m}=\phi_{0}+\left(\phi_{1}-\phi_{0}\right)+\left(\phi_{2}-\phi_{1}\right)+\cdots+\left(\phi_{m}-\phi_{m-1}\right) .
$$


So,

$$
\lim _{m \rightarrow \infty} \phi_{m}(t)=\phi_{0}(t)+\sum_{i=1}^{\infty}\left(\phi_{i}(t)-\phi_{i-1}(t)\right)
$$

provided that (4.4) converges.

Thus, the convergence of the sequence of functions $\phi_{m}(t)$ is equivalent to the convergence of the right-hand side of (4.4).

To prove the latter, we use estimate (4.3), and then apply the Weierstrass $M$-test. The convergence of the series $\sum_{m=0}^{\infty} A^{m} h^{m+1}$ implies the uniform convergence of the series $\sum_{i=1}^{\infty}\left\|\phi_{i}(t)-\phi_{i-1}(t)\right\|$ on $[\theta, \theta+h]$. This implies that the right-hand side of (4.4) is uniform convergent to some function $\phi(t)$. That is, there exists a function $\phi(t)$ such that $\lim _{m \rightarrow \infty} \phi_{m}(t)=\phi(t)$. Obviously, $\phi(t) \in S\left(x_{0}, b\right)$ since $\left\|\phi(t)-x_{0}\right\|=\lim _{m \rightarrow \infty} \| \phi_{m}(t)-$ $x_{0} \| \leq b$.

(iv) The last part of the proof of the existence is to show that $\phi(t)$ is a solution of (4.1).

From the Lipschitz condition,

$$
\left\|f(t, \phi(t))-f\left(t, \phi_{m}(t)\right)\right\| \leq A\left\|\phi(t)-\phi_{m}(t)\right\|
$$

for all $t \in[\theta, \theta+h]$ and $m \in \mathbb{N}$.

Thus, for every $\epsilon>0, \exists n_{0} \in \mathbb{N}$ such that

$$
\left\|f(t, \phi(t))-f\left(t, \phi_{m}(t)\right)\right\|<\frac{\epsilon}{h} \quad \forall m \geq n_{0}, t \in[\theta, \theta+h] .
$$

So,

$$
\begin{aligned}
\| \int_{\theta}^{t}(f(s, \phi(s)))-f\left(s, \phi_{m}(s)\right) d_{q, \omega} s & <\frac{\epsilon}{h} \int_{\theta}^{t} d_{q, \omega} s \\
& \leq \epsilon .
\end{aligned}
$$

This implies that

$$
\lim _{m \rightarrow \infty} \int_{\theta}^{t} f\left(s, \phi_{m}(s)\right) d_{q, \omega} s=\int_{\theta}^{t} f(s, \phi(s)) d_{q, \omega} s
$$

uniformly on $[\theta, \theta+h]$. Taking $k \rightarrow \infty$ in (4.2), it follows that

$$
\phi(t)=x_{0}+\int_{\theta}^{t} f(s, \phi(s)) d_{q, \omega} s .
$$

Consequently, we obtain $D_{q, \omega} \phi(t)=f(t, \phi(t))$. Clearly, $\phi(\theta)=x_{0}$.

\section{Uniqueness}

To prove the uniqueness, let us assume that $\psi(t)$ is another solution which is valid in $t \in[\theta, \theta+h]$. We have

$$
\phi(t)=\phi(h(t))+(t-h(t)) f(t, \phi(t))
$$


and

$$
\psi(t)=\psi(h(t))+(t-h(t)) f(t, \psi(t)) .
$$

From which we deduce

$$
\|\phi(t)-\psi(t)\| \leq\|\phi(h(t))-\psi(h(t))\|+|t-h(t)|\|f(t, \phi(t))-f(t, \psi(t))\| .
$$

Taking $\sigma(t)=\|\phi(t)-\psi(t)\|$, we get

$$
\sigma(t)(1-A|t-h(t)|) \leq \sigma(h(t))
$$

i.e.,

$$
\sigma(t) \leq \sigma(h(t))+A|t-h(t)| \sigma(t)
$$

Hence,

$$
\begin{aligned}
\sigma(t) & \leq \frac{\sigma(h(t))}{1-A|t-h(t)|} \\
& \leq \frac{\sigma\left(h^{k}(t)\right)}{\prod_{i=0}^{k-1}\left(1-A\left|h^{i}(t)-h^{i+1}(t)\right|\right)} \\
& =\frac{\sigma\left(h^{k}(t)\right)}{\prod_{i=0}^{k-1}\left(1-A q^{i}(t(1-q)-\omega)\right)}, \quad k \in \mathbb{N} .
\end{aligned}
$$

By taking the limit as $k \rightarrow \infty$, we get

$$
\sigma(t) \leq \sigma(\theta) e_{A}(t)
$$

Since $\sigma(\theta)=0$, then $\sigma(t)=0$, which completes the proof.

Corollary 4.2 Let I be an interval containing $\theta, f_{i}\left(t, x_{1}, x_{2}, \ldots, x_{n}\right): I \times \prod_{i=1}^{n} S_{i}\left(y_{i}, b_{i}\right) \rightarrow X$ such that the following conditions are satisfied:

(i) For $x_{i} \in S_{i}\left(y_{i}, b_{i}\right), 1 \leq i \leq n, f_{i}\left(t, x_{1}, x_{2}, \ldots, x_{n}\right)$ are continuous at $t=\theta$.

(ii) There is a positive constant $A$ such that, for $t \in I, x_{i}, x_{i}^{\prime} \in S_{i}\left(y_{i}, b_{i}\right), 1 \leq i \leq n$, the following Lipschitz condition is satisfied:

$$
\left\|f_{i}\left(t, x_{1}, x_{2}, \ldots, x_{n}\right)-f_{i}\left(t, x_{1}^{\prime}, x_{2}^{\prime}, \ldots, x_{n}^{\prime}\right)\right\| \leq A \sum_{i=1}^{n}\left\|x_{i}-x_{i}^{\prime}\right\| .
$$

Then there exists a unique solution of the initial value problem

$$
\left.\begin{array}{l}
D_{q, \omega} x_{i}(t)=f_{i}\left(t, x_{1}(t), x_{2}(t), \ldots, x_{n}(t)\right), \quad 1 \leq i \leq n, t \in I, \\
x_{i}(\theta)=y_{i} \in X .
\end{array}\right\}
$$


Proof Let $x_{0}=\left(y_{1}, y_{2}, \ldots, y_{n}\right)^{T}$ and $b=\left(b_{1}, b_{2}, \ldots, b_{n}\right)^{T}$.

Define $f: I \times \prod_{i=1}^{n} S_{i}\left(y_{i}, b_{i}\right) \rightarrow X^{n}$ by

$$
f\left(t, x_{1}, x_{2}, \ldots, x_{n}\right)=\left(f_{1}\left(t, x_{1}, x_{2}, \ldots, x_{n}\right), \ldots, f_{n}\left(t, x_{1}, x_{2}, \ldots, x_{n}\right)\right)^{T} .
$$

System (4.6) yields

$$
\left.\begin{array}{l}
D_{q, \omega} x(t)=f(t, x(t)), \\
x(\theta)=x_{0} .
\end{array}\right\}
$$

First $f$ is continuous at $t=\theta$, since each $f_{i}$ is continuous at $t=\theta$.

Second $f$ satisfies a Lipschitz condition because for $x, x^{\prime} \in \prod_{i=1}^{n} S_{i}\left(y_{i}, b_{i}\right)$

$$
\begin{aligned}
\left\|f(t, x)-f\left(t, x^{\prime}\right)\right\| & =\left\|f\left(t, x_{1}, x_{2}, \ldots, x_{n}\right)-f\left(t, x_{1}^{\prime}, x_{2}^{\prime}, \ldots, x_{n}^{\prime}\right)\right\| \\
& =\sum_{i=1}^{n}\left\|f_{i}\left(t, x_{1}, x_{2}, \ldots, x_{n}\right)-f_{i}\left(t, x_{1}^{\prime}, x_{2}^{\prime}, \ldots, x_{n}^{\prime}\right)\right\| \\
& \leq A \sum_{i=1}^{n}\left\|x_{i}-x_{i}^{\prime}\right\| \\
& =A\left\|x-x^{\prime}\right\| .
\end{aligned}
$$

Applying Theorem 4.1, then there exist $h>0$ such that the initial value problem (4.7) has a unique solution on $[\theta, \theta+h]$. It is easy to show that (4.7) is equivalent to the initial value problem (4.6).

The following corollary shows that Corollary 4.2 can be applied to indicate the required conditions for the existence and uniqueness of solutions of the Cauchy problem

$$
\left.\begin{array}{l}
D_{q, \omega}^{n} x(t)=f\left(t, x(t), D_{q, \omega} x(t), \ldots, D_{q, \omega}^{n-1} x(t)\right), \\
D_{q, \omega}^{i-1} x(\theta)=y_{i}, \quad 1 \leq i \leq n .
\end{array}\right\}
$$

The proof of this corollary depends on converting the $n$th order Hahn difference equation (4.8) to a first order system. Clearly, the Cauchy problem (4.8) is equivalent to the first order system

$$
\left.\begin{array}{l}
D_{q, \omega} x_{i}(t)=f_{i}\left(t, x_{1}(t), x_{2}(t), \ldots, x_{n}(t)\right), \quad 1 \leq i \leq n, \\
x_{i}(\theta)=y_{i}
\end{array}\right\}
$$

in the sense that $\left\{\phi_{i}(t)\right\}_{i=1}^{n}$ is a solution of (4.9) if and only if $\phi_{1}(t)$ is a solution of (4.8). Here,

$$
f_{i}\left(t, x_{1}, \ldots, x_{n}\right)= \begin{cases}x_{i+1}, & 1 \leq i \leq n-1 \\ f\left(t, x_{1}, \ldots, x_{n}\right), & i=n\end{cases}
$$

This leads us to state the following theorem.

Theorem 4.3 Let $f\left(t, x_{1}, \ldots, x_{n}\right)$ be a function defined on $I \times \prod_{i=1}^{n} S_{i}\left(y_{i}, b_{i}\right)$ such that the following conditions are satisfied: 
(i) For any values of $x_{r} \in S_{r}\left(y_{r}, b_{r}\right), f$ is continuous at $t=\theta$.

(ii) $f$ satisfies a Lipschitz condition

$$
\left\|f\left(t, x_{1}, \ldots, x_{n}\right)-f\left(t, x_{1}^{\prime}, \ldots, x_{n}^{\prime}\right)\right\| \leq A \sum_{i=1}^{n}\left\|x_{i}-x_{i}^{\prime}\right\|,
$$

where $A>0, x_{i}, x_{i}^{\prime} \in S_{i}\left(y_{i}, b_{i}\right), i=1, \ldots, n$ and $t \in I$.

Then the Cauchy problem (4.8) has a unique solution which is valid on $[\theta, \theta+h]$.

The following corollary gives us the required conditions for the existence of solutions of the Cauchy problem

$$
\left.\begin{array}{l}
a_{0}(t) D_{q, \omega}^{n} x(t)+a_{1}(t) D_{q, \omega}^{n-1} x(t)+\cdots+a_{n}(t) x(t)=b(t) \\
D_{q, \omega}^{i-1} x(\theta)=y_{i}, \quad i=1, \ldots, n
\end{array}\right\}
$$

Corollary 4.4 Assume that the functions $a_{j}(t): I \rightarrow \mathbb{C}(0 \leq j \leq n)$ and $b(t): I \rightarrow X$ satisfy the following conditions:

(i) $a_{j}(t)(j=1, \ldots, n)$ and $b(t)$ are continuous at $\theta$ with $a_{0}(t) \neq 0 \forall t \in I$.

(ii) $a_{j}(t) / a_{0}(t)$ is bounded on $I, j \in\{1, \ldots, n\}$.

Then, for any elements $y_{r} \in X$, equation (4.10) has a unique solution on a subinterval $J \subset I$ containing $\theta$.

Proof Dividing by $a_{0}(t)$, we get the equation

$$
D_{q, \omega}^{n} x(t)=A_{1}(t) D_{q, \omega}^{n-1} x(t)+\cdots+A_{n}(t) x(t)+B(t),
$$

where $A_{j}(t)=-a_{j}(t) / a_{0}(t)$, and $B(t)=b(t) / a_{0}(t)$. Since $A_{j}(t)$ and $B(t)$ are continuous at $t=\theta$, then the function $f\left(t, x_{1}, x_{2}, \ldots, x_{n}\right)$ defined by

$$
f\left(t, x_{1}, x_{2}, \ldots, x_{n}\right)=A_{1}(t) x_{n}+\cdots+A_{n}(t) x_{1}+B(t)
$$

is continuous at $t=\theta$. Furthermore, $A_{j}(t)$ is bounded on $I$. Consequently, there is $A>0$ such that $\left|A_{j}(t)\right| \leq A, t \in I$. We can easily see that $f$ satisfies a Lipschitz condition with Lipschitz constant $A$. Thus, the function $f\left(t, x_{1}, \ldots, x_{n}\right)$ satisfies the conditions of Theorem 4.3. Hence, there exists a unique solution of (4.11) on a subinterval $J$ of $I$ containing $\theta$.

\section{Nonlocal results}

Theorem 4.1 is called a local existence theorem, because it guarantees the existence of a solution $x(t)$ defined for $t \in R$, which is close to the initial point $\theta$. However, in many situations, a solution will actually exist on the entire interval $I=[\theta, \theta+a]$. We now show that if $f$ satisfies a Lipschitz condition on a strip

$$
S=[\theta, \theta+a] \times X
$$

rather than on the rectangle $R$, which is given in Section 4, then solutions will exist on the entire interval $I=[\theta, \theta+a]$. 
Theorem 5.1 Let $f$ be continuous on the strip $S$, and suppose that there exists a constant $A>0$ such that $\|f(t, x)-f(t, y)\| \leq A\|x-y\|$ for all $(t, x),(t, y) \in S$, where $A<\frac{1}{a(1-q)}$. Then the successive approximations that are given in (4.2) exist on the entire interval $[\theta, \theta+a]$ and converge there uniformly to the unique solution of (4.1).

Proof There is a constant $M$ such that $\left\|f\left(t, x_{0}\right)\right\| \leq M$ for all $t \in I$. We will show that the inequality

$$
\left\|\phi_{k}(t)-\phi_{k-1}(t)\right\| \leq M A^{k-1} \frac{(t(1-q)-\omega)^{k}}{(q ; q)_{k}}, \quad t \in I
$$

holds for every $k \in N$. Inequality (5.1) is true at $k=1$. Indeed, we have

$$
\begin{aligned}
\left\|\phi_{1}(t)-\phi_{0}(t)\right\| & =\left\|\int_{\theta}^{t} f\left(s, x_{0}\right) d_{q, \omega} s\right\| \leq M \int_{\theta}^{t} d_{q, \omega} s \\
& =M(t(1-q)-\omega) \frac{1}{1-q}
\end{aligned}
$$

and

$$
\begin{aligned}
\left\|\phi_{2}(t)-\phi_{1}(t)\right\| & =\left\|\int_{\theta}^{t}\left(f\left(s, \phi_{1}(s)\right)-f\left(s, \phi_{0}(s)\right)\right) d_{q, \omega} s\right\| \\
& \leq A \int_{\theta}^{t}\left\|\phi_{1}(s)-\phi_{0}(s)\right\| d_{q, \omega} s \\
& \leq \frac{M A}{1-q} \int_{\theta}^{t}(s(1-q)-\omega) d_{q, \omega} s \\
& =\frac{M A}{1-q}(t(1-q)-\omega)^{2} \frac{1}{1-q^{2}} \\
& =M A \frac{(t(1-q)-\omega)^{2}}{(q ; q)_{2}} .
\end{aligned}
$$

Assume that inequality (5.1) is true. Now, we have

$$
\begin{aligned}
\left\|\phi_{k+1}(t)-\phi_{k}(t)\right\| & \leq \int_{\theta}^{t}\left\|\left(f\left(s, \phi_{k}(s)\right)-f\left(s, \phi_{k-1}(s)\right)\right)\right\| d_{q, \omega} s \\
& \leq \frac{M A^{k}}{(q ; q)_{k}} \int_{\theta}^{t}(s(1-q)-\omega)^{k} d_{q, \omega} s \\
& =\frac{M A^{k}}{(q ; q)_{k}}(t(1-q)-\omega) \sum_{i=0}^{\infty} q^{i}\left(h^{i}(t)(1-q)-\omega\right)^{k} \\
& =\frac{M A^{k}}{(q ; q)_{k+1}}(t(1-q)-\omega)^{k+1} .
\end{aligned}
$$

We see that

$$
\sum_{i=1}^{\infty} \frac{M A^{i-1}(t(1-q)-\omega)^{i}}{(q ; q)_{i}}=\frac{M}{A}\left(\sum_{i=0}^{\infty} \frac{(A(t(1-q)-\omega))^{i}}{(q ; q)_{i}}-1\right)
$$


which converges to $(M / A)\left(e_{A}(t)-1\right), t \in I$. This convergence enables us to apply the Weierstrass $M$-test to conclude that a series

$$
\sum_{k=1}^{\infty}\left\|\phi_{i}(t)-\phi_{i-1}(t)\right\|
$$

converges uniformly on $I$. Also, we can write $\phi_{k}(t)$ as the following sum

$$
\phi_{k}(t)=\phi_{0}(t)+\sum_{i=1}^{k}\left(\phi_{i}(t)-\phi_{i-1}(t)\right) .
$$

Consequently, the absolute uniform convergence of the series in (5.3) on $I$ implies that $\phi_{k}(t)$ converges uniformly to some function $\phi(t)$ on $I$.

Our objective now is to show that $\phi(t)$ is a solution of (4.1) for all $t \in I$.

From the Lipschitz condition

$$
\left\|f(t, \phi(t))-f\left(t, \phi_{m}(t)\right)\right\| \leq A\left\|\phi(t)-\phi_{m}(t)\right\|
$$

for all $t \in[\theta, \theta+a]$. This implies that $\lim _{m \rightarrow \infty} f\left(t, \phi_{m}(t)\right)=f(t, \phi(t))$ uniformly on $I$ and $\lim _{m \rightarrow \infty} \int_{\theta}^{t} f\left(s, \phi_{m}(s)\right) d_{q, \omega} s=\int_{\theta}^{t} f(s, \phi(s)) d_{q, \omega} s$ uniformly on $I$.

Taking $m \rightarrow \infty$ in (4.2), it follows that

$$
\phi(t)=x_{0}+\int_{\theta}^{t} f(s, \phi(s)) d_{q, \omega} s
$$

Consequently, $D_{q, \omega} \phi(t)=f(t, \phi(t))$. Clearly, $\phi(\theta)=x_{0}$.

\section{Uniqueness}

Let $x(t)$ and $y(t)$ be two solutions of (4.1) for all $t \in I$. We show that $x(t) \equiv y(t)$ on $I$.

For $t \in I$, consider

$$
\begin{aligned}
z(t) & =\|x(t)-y(t)\| \\
& \leq \int_{\theta}^{t}\|f(s, x(s))-f(s, y(s))\| d_{q, \omega} s \\
& \leq A \int_{\theta}^{t}\|x(s)-y(s)\| d_{q, \omega} s \\
& =A \int_{\theta}^{t} z(s) d_{q, \omega} s .
\end{aligned}
$$

Thus, from Gronwall's inequality, we have $z(t)=0$. Consequently, $x(t)=y(t)$ for all $t \in I$.

\section{Corollary 5.2 Let $f$ be continuous on the half-plane}

$$
[\theta, \infty) \times X
$$

Assume that $f$ satisfies a Lipschitz condition

$$
\|f(t, x)-f(t, y)\| \leq L_{\theta, a}\|x-y\|
$$


on each strip

$$
S_{\theta, a}=[\theta, \theta+a] \times X,
$$

where $L_{\theta, a}$ is a constant that may depend on $\theta$ and $a$. Then, the initial value problem (4.1) has a unique solution that exists on the whole half-line $[\theta, \infty)$.

Proof The proof involves showing that the conditions of Theorem 5.1 hold on every strip of $S_{\theta, a}$ and is omitted for brevity.

\section{Conclusion and future directions}

This article was devoted to establish the method of successive approximations in proving the existence and uniqueness of solutions of the initial value problems associated with Hahn difference operators. Also, some new results of the calculus based on this operator like a mean value theorem were obtained. In one direction, one should ask about the $q-\omega$ Taylor's theorem. In this respect, we point out that $q$-Taylor's theorem has been established in [5]. Another direction, is to study in more details the theory of Hahn difference equations, based on Hahn difference operator, and the stability of its solutions.

\section{Competing interests}

The authors declare that they have no competing interests.

Authors' contributions

All authors contributed equally in writing this paper. All authors read and approved the final manuscript.

\section{Author details}

${ }^{1}$ Department of Mathematics, Faculty of Science, Cairo University, Giza, Egypt. ${ }^{2}$ Mathematics Department, Faculty of Science, Al-Azhar University (Girls Branch), Nasr City, Cairo, Egypt

Received: 25 March 2013 Accepted: 4 September 2013 Published: 08 Nov 2013

\section{References}

1. Hahn, W: Über orthogonalpolynome, die q-differenzengleichungen genügen. Math. Nachr. 2, 4-34 (1949)

2. Hahn, W: Ein Beitrag zur Theorie der Orthogonalpolynome. Monatshefte Math. 95, 19-24 (1983)

3. Abu Risha, MH, Annaby, MH, Ismail, MEH, Mansour, ZS: Linear q-difference equations. Z. Anal. Anwend. 26, 481-494 (2007)

4. Andrews, GE, Askey, R, Roy, R: Special Functions. Cambridge University Press, Cambridge (1999)

5. Annaby, MH, Mansour, ZS: q-Taylor and interpolation series for Jackson q-difference operator. J. Math. Anal. Appl. 344(1), 472-483 (2008)

6. Carmichael, RD: Linear difference equations and their analytic solutions. Trans. Am. Math. Soc. 12, $99-134$ (1911)

7. Carmichael, RD: On the theory of linear difference equations. Am. J. Math. 35, 163-182 (1913)

8. Ismail, MEH: Classical and Quantum Orthogonal Polynomials in One Variable. Encyclopedia Math. Appl., vol. 98. Cambridge University Press, Cambridge (2005)

9. Jackson, FH: Basic integration. Q. J. Math. 2, 1-16 (1951)

10. Jackson, FH: On q-functions and a certain difference operator. Trans. R. Soc. Edinb. 46, 253-281 (1908)

11. Bird, MT: On generalizations of sum formulas of the Euler-Maclaurin type. Am. J. Math. 58, 487-503 (1936)

12. Birkhoff, GD: General theory of linear difference equations. Trans. Am. Math. Soc. 12, 243-284 (1911)

13. Jagerman, DL: Difference Equations with Applications to Queues. Dekker, New York (2000)

14. Jordan, C: Calculus of Finite Differences. Chelsea, New York (1965)

15. Annaby, MH, Hamza, AE, Aldwoah, KA: Hahn difference operator and associated Jackson-Nörlund integrals. J. Optim. Theory Appl. 154, 133-153 (2012)

16. Abu Risha, MH, Annaby, MH, Ismail, MEH, Mansour, ZS: Existence and uniqueness theorems of q-difference equations (2005, submitted)

17. Koornwinder, TH: Special functions and q-commuting variables. In: Ismail, MEH, Masson, DR, Rahman, M (eds.) Special Functions, q-Series and Related Topics. Fields Institute Communications, vol. 14, pp. 131-166. Am. Math. Soc., Providence (1997)

18. Gasper, G, Rahman, M: Basic Hypergeometric Series, 2nd edn. Cambridge University Press, Cambridge, (2004)

19. Mahwin, J: Boundary value problems for nonlinear ordinary differential equations: from successive approximations to topology. In: Development of Mathematics 1900-1950 (Luxembourg, 1992), pp. 443-477. Birkhäuser, Basel (1994)

20. Picard, E: Mémoire sur la théorie des équations aux dérivés partielles et la méthode des approximations successives. J. Math. Pures Appl. 6, 145-210 (1890) 
10.1186/1687-1847-2013-316

Cite this article as: Hamza and Ahmed: Existence and uniqueness of solutions of Hahn difference equations. Advances in Difference Equations 2013, 2013:316

Submit your manuscript to a SpringerOpen ${ }^{\circ}$ journal and benefit from:

- Convenient online submission

- Rigorous peer review

- Immediate publication on acceptance

Open access: articles freely available online

- High visibility within the field

- Retaining the copyright to your article

Submit your next manuscript at $\gg$ springeropen.com 